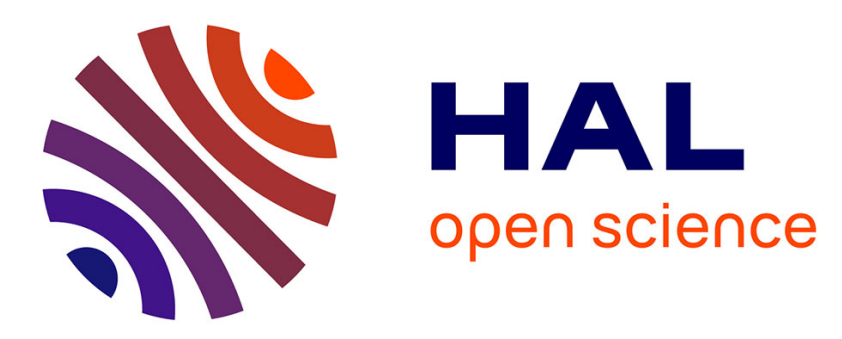

\title{
Mid-infrared extension of supercontinuum in chalcogenide suspended core fibre through soliton gas pumping
}

Julien Fatome, Bertrand Kibler, Mohammed El-Amraoui, Jean-Charles Jules, Grégory Gadret, Frédéric Desevedavy, Frédéric Smektala

\section{To cite this version:}

Julien Fatome, Bertrand Kibler, Mohammed El-Amraoui, Jean-Charles Jules, Grégory Gadret, et al.. Mid-infrared extension of supercontinuum in chalcogenide suspended core fibre through soliton gas pumping. Electronics Letters, 2011, 47 (6), pp.398-400. 10.1049/el.2010.7520 . hal-00577037

\section{HAL Id: hal-00577037 \\ https://hal.science/hal-00577037}

Submitted on 16 Mar 2011

HAL is a multi-disciplinary open access archive for the deposit and dissemination of scientific research documents, whether they are published or not. The documents may come from teaching and research institutions in France or abroad, or from public or private research centers.
L'archive ouverte pluridisciplinaire HAL, est destinée au dépôt et à la diffusion de documents scientifiques de niveau recherche, publiés ou non, émanant des établissements d'enseignement et de recherche français ou étrangers, des laboratoires publics ou privés. 


\section{Mid-infrared extension of supercontinuum in a chalcogenide suspended core fibre through soliton gas pumping}

J. Fatome, B. Kibler, M. El-Amraoui, J-C. Jules, G. Gadret, F. Desevedavy and F.

Smektala

\section{Authors' affiliations:}

J. Fatome, B. Kibler, M. El-Amraoui, J-C. Jules, G. Gadret, F. Desevedavy and F. Smektala

Laboratoire Interdisciplinaire Carnot de Bourgogne, UMR 5209 CNRS-Université de Bourgogne, 21078 DIJON, FRANCE.

Contact Author: J. Fatome

Contact Address: Laboratoire Interdisciplinaire Carnot de Bourgogne, UMR 5209 CNRS-Université de Bourgogne

9 Avenue Alain Savary, BP 47870

21078 Dijon

FRANCE

Fax: $\quad$ +33 $\quad$ (0)3 80395971

Phone: $\quad$ +33 (0)3 80395995

Email: $\quad$ julien.fatome@u-bourgogne.fr 


\begin{abstract}
We experimentally demonstrate the mid-infrared extension of supercontinuum in a $1.9-\mu \mathrm{m}$ suspended core chalcogenide fibre whose zero dispersion wavelength is shifted to 1.9 $\mu \mathrm{m}$, i.e., into the transparency window of standard silica fibres. Based on the well-known long-pulse regime of supercontinuum generation in silica fibre, we are able to convert and shift a low-cost optical quasi-CW source at $1.53 \mu \mathrm{m}$ into a large number of interacting femtosecond solitons localized up to $2.1 \mu \mathrm{m}$. This optical source called a soliton gas allows us to pump a $50-\mathrm{cm}$ long chalcogenide microstructured fibre near its zero dispersion wavelength, thus leading to an efficient extension of a mid-infrared supercontinuum beyond $2.4 \mu \mathrm{m}$.
\end{abstract}


Introduction: Fibre-based supercontinuum (SC) sources in the mid-infrared (mid-IR) have a great potential for many applications such as optical frequency metrology, optical tomography and spectroscopy. It is well known that soft-glass fibres are promising candidates for such devices due to their low mid-IR losses and high intrinsic nonlinearities in opposition to silica [1]. However, the zero dispersion wavelengths (ZDW) of these materials are generally beyond $2 \mu \mathrm{m}$ and far from the wavelengths of conventional fibrebased laser sources. Consequently, the use of non-silica fibres often requires costly and high power pump lasers to achieve an efficient spectral broadening up to the mid-IR. During the last decade, research activities have been focused on the development of microstructured optical fibre (MOF) technology for dispersion-tailored waveguides as well as shifted near-infrared lasers to longer wavelengths so as to pump the fibre under test close to its ZDW [1]. In this Letter, we propose to combine these both attractive options by using the Raman soliton self-frequency-shift process (SSFS) occurring around $1550 \mathrm{~nm}$ in a highly nonlinear silica fiber (HNLF) combined with a chalcogenide suspended core fiber which ZDW has been shifted from $4.8 \mu \mathrm{m}$ to $1.9 \mu \mathrm{m}$ through its MOF profile in order to extend a SC in the mid-IR region beyond $2.4 \mu \mathrm{m}$.

Experimental configuration: The experimental set-up is sketched in Fig. 1 and is basically made of two stages cascaded SC generation towards the mid-IR. The first stage shifts the laser frequency through Raman SSFS which is commonly used to shift ultrafast 1550-nm pulses to lower frequencies in silica fibers [2,3]. To this end, we use a low-cost and extremely compact commercial high-power sub-nanosecond pump. Indeed, our passively Q-switched micro-laser delivers $3.7 \mathrm{~ns}$ pulses at $1535 \mathrm{~nm}$, with an average power around $19 \mathrm{~mW}$ and a pulse repetition rate of $2.7 \mathrm{kHz}$, corresponding to pulse energy of $7.5 \mu \mathrm{J}$ and peak power up to $2 \mathrm{~kW}$. Based on the well-known long-pulse regime of SC generation in silica fibre [4], we take advantage both of modulation instability (MI) and 
Raman SSFS processes to convert our quasi-continuous-wave (CW) source at $1.53 \mu \mathrm{m}$ into a large number of interacting femtosecond solitons around $2 \mu \mathrm{m}$. The fibre is a $50-\mathrm{m}$ long segment of highly nonlinear silica fibre (HNLF) characterized by a chromatic dispersion parameter $D=0.6 \mathrm{ps} / \mathrm{km} / \mathrm{nm}$ and a nonlinear Kerr coefficient $\gamma=10 \mathrm{~W}^{-1} \cdot \mathrm{km}^{-1}$ at the laser frequency. The fibre exhibits a low dispersion slope $D_{S}=0.007 \mathrm{ps} / \mathrm{km} / \mathrm{nm}^{2}$ and losses around $0.6 \mathrm{~dB} / \mathrm{km}$ at $1.55 \mu \mathrm{m}$. By using a simple 20x microscope objective (MO) we reach about $40 \%$ of coupling efficiency into the HNLF. In this configuration, the SC formation begins with the $\mathrm{Ml}$ of the continuous pump wave in the anomalous dispersion region, thus evolving into a train of ultra-short soliton pulses [4,5]. Since $\mathrm{Ml}$ is a noisy seeded process, the generated solitons will have variable bandwidths and energies. Based on the fibre parameters and the input pump power, we calculate the maximum MI gain at a frequency $\Omega_{\max }=22.6 \mathrm{THz}$ [6]. In the time domain, the $\mathrm{CW}$ is thus converted into a periodic pulse train characterized by a period $T=2 \pi / \Omega_{\max }=45 \mathrm{fs}$. The soliton pulses therefore exhibit a temporal width below the period value. Their bandwidth is large enough for intra-pulse Raman scattering to occur leading to a continuous red-shift along the HNLF. Moreover, the different peak powers create a large range of self-frequency shifts, creating a smooth red-shifted continuum [5,7] (see also next section). This specific spreading in time and frequency domains of the large number of interacting solitons has been already related to the idea of the soliton gas [5]. The frequency shift of the soliton gas is a function of the HNLF length used and it can be suitably adjusted in order to pump the chalcogenide MOF as close as possible to the ZDW, as shown in the next section. Note that the long wavelength extent of the HNLF continuum will be limited here by the high losses of silica glass beyond $2.1 \mu \mathrm{m}$.

In order to extend the SC in the mid-IR, the second stage of our setup (see Fig. 1) consists in injecting the resulting HNLF soliton gas into a $50-\mathrm{cm}$ long segment of chalcogenide MOF designed with a suspended core made of $\mathrm{As}_{2} \mathrm{~S}_{3}$ glass. The input signal was coupled into the chalcogenide fiber by means of the alignment option of an optical fiber splicing device combined with a 3- $\mu$ m waist micro-lens fibre, which allows an efficient fiber coupling up to $30-40 \%$ [8]. 
Figure 2(a) shows the corresponding SEM image of the fibre cross section. Here, the main idea is to use the attractive dispersive and nonlinear characteristics of this small core MOF with a diameter close to $1.9 \mu \mathrm{m}$. The calculated dispersion curve of the suspended core MOF is presented in Fig. 2(b) and the ZDW is predicted at wavelengths close to $1.9 \mu \mathrm{m}$ by taking into account $10 \%$ discrepancies on the fibre core size. From our mode calculations, we have also deduced an effective mode area about $2.4 \mu \mathrm{m}^{2}$ at the ZDW, corresponding to an estimated nonlinear Kerr coefficient $\gamma=3.86 \mathrm{~W}^{-1} \cdot \mathrm{m}^{-1}$ with $\mathrm{n}_{2}=$ $2.810^{-18} \mathrm{~m}^{2} / \mathrm{W}$ [8]. Recently, we have also reported in a chalcogenide MOF that Raman gain is 180 larger than fused silica [9], which underlines the potential of this kind of fiber combined with anomalous dispersion for the SSFS process. We thus expect that pumping our suspended core MOF with the soliton gas source in the anomalous dispersion regime will efficiently extend the SC towards the mid-IR by using very short lengths of fibre. Note that our chalcogenide fibre exhibits a large transparency bandwidth in the mid-IR with typical losses about $0.5 \mathrm{~dB} / \mathrm{m}$ measured in the wavelength range studied [8]. However, extrinsic losses due to impurity absorption (i.e., OH-absorption) affect the glass transmission which can result in high loss absorption peaks at particular wavelengths such as 1.4 and $1.9 \mu \mathrm{m}$, see Fig. 3 and experimental results section.

Results and discussion: The silica and chalcogenide fibers are then chosen and arranged with the final aim to achieve the most significant spectral broadening towards the mid-IR. Figure 3(a) first depicts the experimental results recorded at the output of the HNLF fiber for 4 different lengths of fiber. As expected, the MI of the continuous pump wave evolving into ultrashort soliton pulses induces intra-pulse Raman self-frequency shifts and subsequently a smooth SC generation. As the length of the HNLF increases, we clearly observe an accumulation of variable bandwidths and energies pulses at the leading edge of the SC qualified as soliton gas [5]. By carefully adjusting the length of the HNLF between 20 and $50 \mathrm{~m}$, we can then localize this soliton gas around $2 \mu \mathrm{m}$ in a range of 200 $\mathrm{nm}$. Beyond $50 \mathrm{~m}$, the induced SSFS of the soliton gas reaches the transparency limit of the silica fiber, leading to a rapid decrease of its available energy. Note that the high- 
frequency part of the SC observed in the HNLF below $1.4 \mu \mathrm{m}$ in Fig. 3 is generated through dispersive wave formation in the normal dispersion regime. Indeed, the Mlinduced solitons are spectrally broad enough such that they overlap with the normal dispersion region of the HNLF (ZDW $1.4 \mu \mathrm{m}$ ), and can excite dispersive waves at phase-matched wavelengths $[2,3]$.

When now pumping the $50-\mathrm{cm}$ long chalcogenide MOF with the different soliton gas described above (Fig. 3(b)), we clearly observe that the SSFS phenomenon starts again so as to extend the initial SC beyond $2.4 \mu \mathrm{m}$ (limit of our OSA), which proves that the chalcogenide fiber is pumped into its anomalous dispersion regime. Note that for the $10-\mathrm{m}$ long HNLF configuration, the residual energy contained inside the 1535-nm nanosecond pump is then sufficiently high to generate two spontaneous Raman stokes components in the chalcogenide MOF (Fig. 3(b), lightest grey solid line). Finally, the optimum SC extension in the chalcogenide MOF beyond $2.4 \mu \mathrm{m}$ was obtained for a HNLF length of 30 $\mathrm{m}$ and an associated soliton gas localized around $2 \mu \mathrm{m}$, which could be interpreted as a ZDW below $2 \mu \mathrm{m}$ for the Chalcogenide fiber. Indeed, this is confirmed by the fact that the $\mathrm{SC}$ begins its extension by pumping with the soliton gas localized over the range 1.8-2 $\mu \mathrm{m}$ (10-m-long segment of HNLF).

Conclusions: In conclusion, we have shown that it is possible to extend a silica-based supercontinuum beyond $2.4 \mu \mathrm{m}$ through the generation of a soliton gas inside a HNLF and its injection beyond the ZDW in a $50-\mathrm{cm}$ long chalcogenide suspended core MOF. These results reveal that the concatenation of suitably designed optical fibers, in particular based on different materials, could be an attractive solution in order to generate and extend SC sources in the mid-IR region and especially far beyond the silica transparency limit. 
Acknowledgements: This research was supported by the Agence Nationale de la Recherche (FENOPTIC ANR-09-NANO-023-04 and Confian project) and the Conseil Regional de Bourgogne. We thank Yokogawa and Absys for equipment support. We also thank I. Skripatchev, C. Plocchini, and Y. Messaddeq (Institute of Chemistry, Araraquara, Brazil) for their help on the drawing process. 


\section{References}

1. J. M. Dudley and J. R. Taylor, Supercontinuum generation in optical fiber (Cambridge University Press, 2010).

2. C. Xia, M. Kumar, O. P. Kulkarni, M. N. Islam, F. L. Terry, M. J. Freeman, M. Poulain, G. Mazé, "Mid-infrared supercontinuum generation to $4.5 \mu \mathrm{m}$ in ZBLAN fluoride fibers by nanosecond diode pumping," Optics Letters 31, 2553-2555 (2006).

3. C. L. Hagen, J. W. Walewski, S. T. Sanders, "Generation of a continuum extending to the midinfrared by pumping ZBLAN fiber with an ultrafast 1550-nm source," IEEE Photonics Technology Letters 18, 91-93 (2006).

4. J. M. Dudley, G. Genty, S. Coen, "Supercontinuum generation in photonic crystal fiber," Reviews of Modern Physics 78, 1135-1184 (2006).

5. J. C. Travers, "Blue solitary waves from infrared continuous wave pumping of optical fibers," Optics Express 17, 1502-1507 (2009).

6. G. P. Agrawal, Nonlinear Fiber Optics, 3th Ed (Academic Press, Boston, 2001).

7. C. Xia, M. Kumar, M.-Y. Cheng, O. P. Kulkarni, M. N. Islam, A. Galvanauskas, F. L. Terry, M. J. Freeman, D. A. Nolan, W. A. Wood, "Supercontinuum generation in silica fibers by amplified nanosecond laser diode pulses," IEEE Journal of Selected Topics in Quantum Electronics 13, 789-797 (2007).

8. M. El-Amraoui, J. Fatome, J. C. Jules, B. Kibler, G. Gadret, C. Fortier, F. Smektala, I. Skripatchev, C.F. Polacchini, Y. Messaddeq, J. Troles, L. Brilland, M. Szpulak, G. Renversez, "Strong infrared spectral broadening in low-loss As-S chalcogenide suspended core microstructured optical fibers," Optics Express 18, 4547-4556 (2010).

9. J. Fatome, C. Fortier, T. N. Nguyen, T. Chartier, F. Smektala, K. Messaad, B. Kibler, S. Pitois, G. Gadret, C. Finot, J. Troles, F. Desevedavy, P. Houizot, G. Renversez, L. 
Brilland, N. Traynor, "Linear and nonlinear characterizations of chalcogenide photonic crystal fibers," Journal of Lightwave Technology 27, 1707-1715 (2009). 
Figure Captions:

Fig. 1 Experimental set-up of the cascaded supercontinuum generation in the midinfrared via soliton gas pumping.

Fig. 2 (a) SEM image of the cross section of the chalcogenide microstructured fibre and magnification of the suspended core in the inset. (b) MOF dispersion curves calculated from the microstructure parameters obtained from the SEM picture with $\pm 10 \%$ accuracy on the fibre core size.

Fig. 3 (a) Generation of the soliton gas at the output of the HNLF as a function of fiber length, (b) Corresponding supercontinuum extension after soliton gas injection into the $50-\mathrm{cm}$ long Chalcogenide MOF as a function of initial HNLF length. 


\section{FIGURE 1}

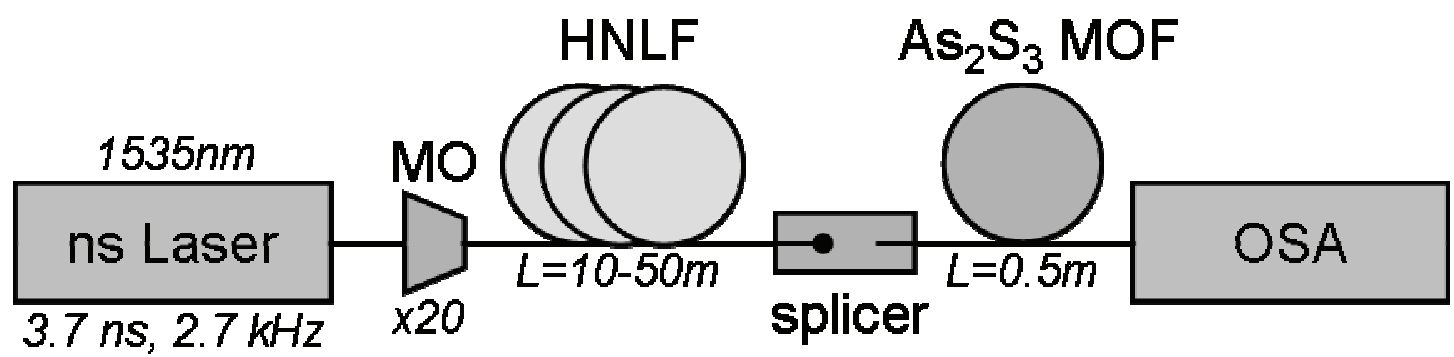

Fig. 1 Experimental set-up of the cascaded supercontinuum generation in the mid-infrared via soliton gas pumping. 
FIGURE 2
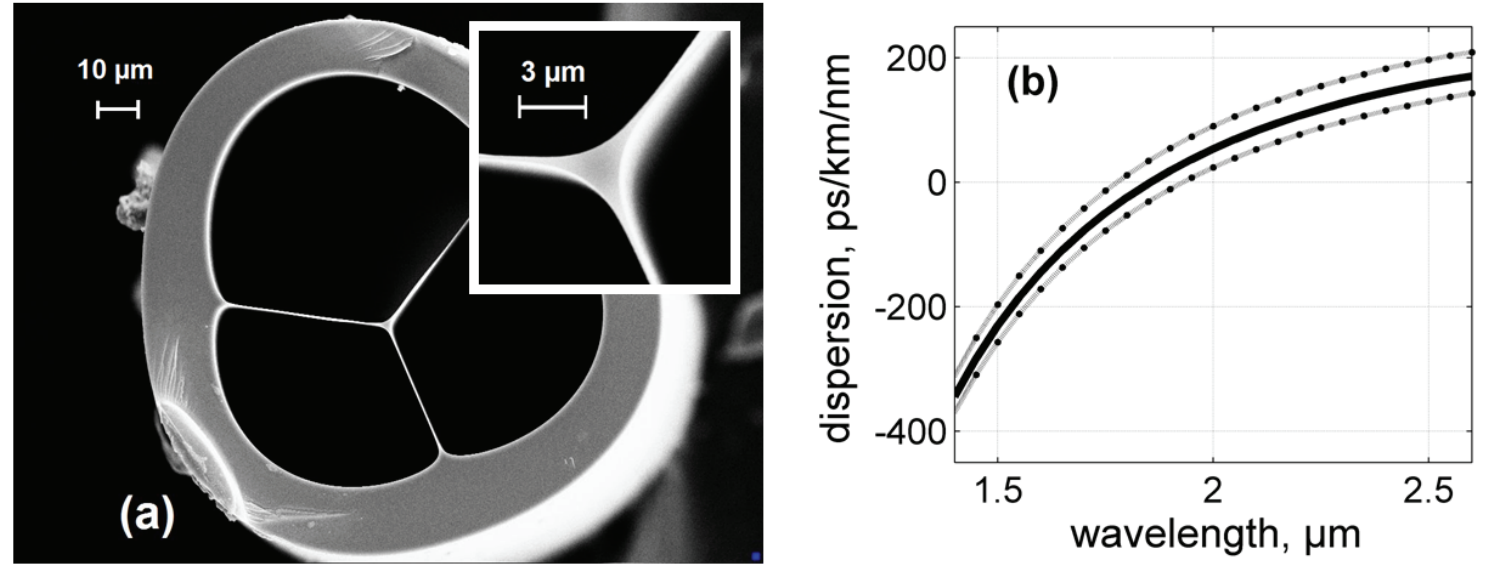

Fig. 2 (a) SEM image of the cross section of the chalcogenide microstructured fibre and magnification of the suspended core in the inset. (b) MOF dispersion curves calculated from the microstructure parameters obtained from the SEM picture with $\pm 10 \%$ accuracy on the fibre core size. 
FIGURE 3
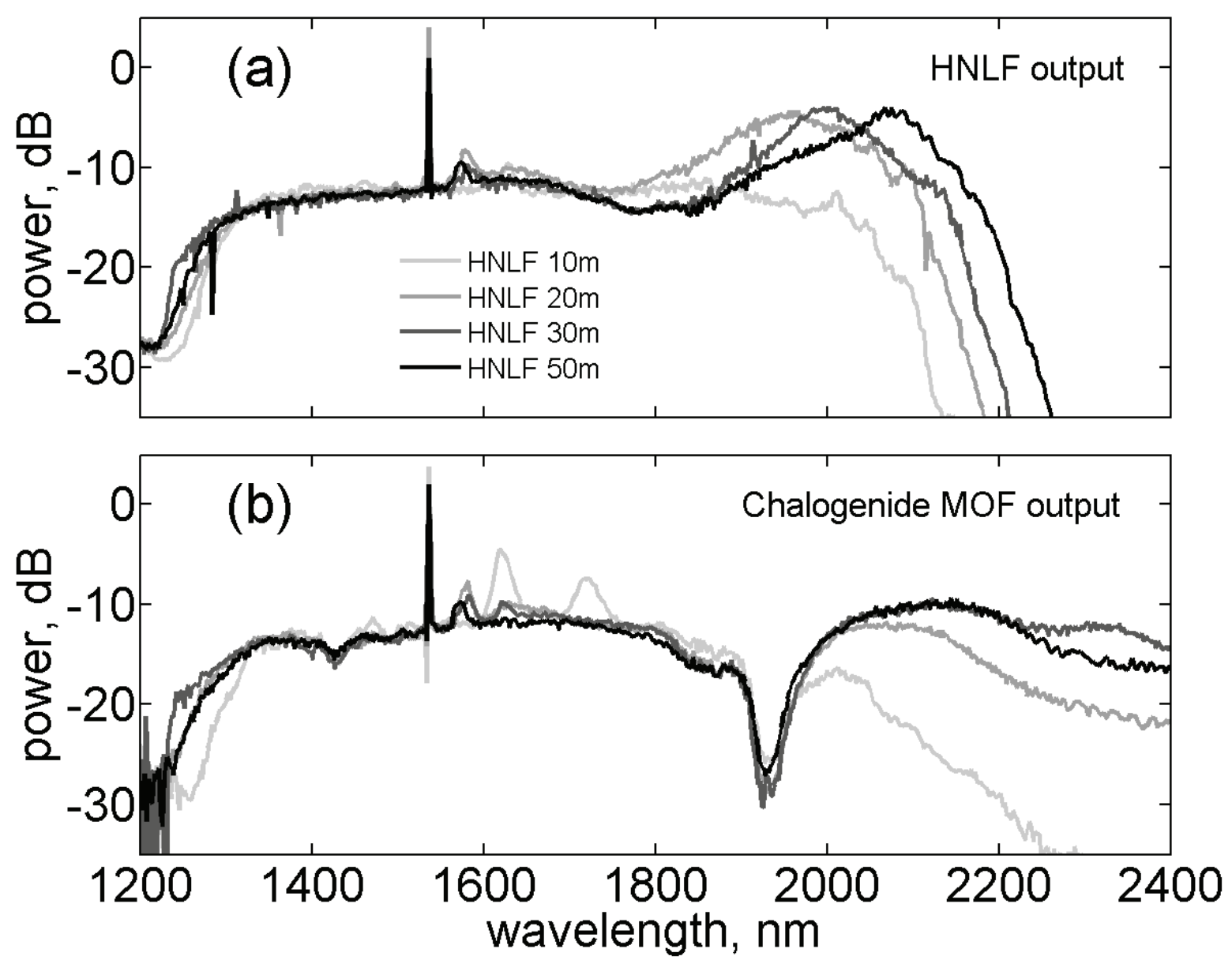

Fig. 3 (a) Generation of the soliton gas at the output of the HNLF as a function of fiber length, (b) Corresponding supercontinuum extension after soliton gas injection into the $50-\mathrm{cm}$ long Chalcogenide MOF as a function of initial HNLF length. 